\title{
Intraoperative cardiac arrest caused by air embolism during video-assisted thoracoscopic segmentectomy
}

\author{
Nai-Liang Li, MD, ${ }^{\text {a }}$ Chen-Sung Lin, MD, PhD, ${ }^{\text {b,c }}$ Chih-Hsun Shih, MD, ${ }^{b}$ and Chia-Chuan Liu, MD, ${ }^{b}$ \\ Taichung City, Taiwan
}

\footnotetext{
From the ${ }^{\mathrm{a} D e p a r t m e n t}$ of Anesthesiology, and ${ }^{\mathrm{b}}$ Thoracic Surgery, Department of Surgery, Koo Foundation Sun Yat-Sen Cancer Center; and ${ }^{\mathrm{c}}$ Thoracic Surgery, Feng-Yuan Hospital, Ministry of Health and Welfare, Taichung City, Taiwan.

Disclosures: Authors have nothing to disclose with regard to commercial support.

Received for publication Aug 24, 2017; revisions received Oct 26, 2017; accepted for publication Nov 9, 2017; available ahead of print Dec 13, 2017.

Address for reprints: Chia-Chuan Liu, MD, 125 Lih-Der Rd, 11152 Taipei, Taiwan, R.O.C (E-mail: gcliu@ kfsyscc.org).

J Thorac Cardiovasc Surg 2018;155:e111-3

$0022-5223 / \$ 36.00$

Copyright (C) 2017 Published by Elsevier Inc. on behalf of The American Association for Thoracic Surgery

https://doi.org/10.1016/j.jtcvs.2017.11.015
}

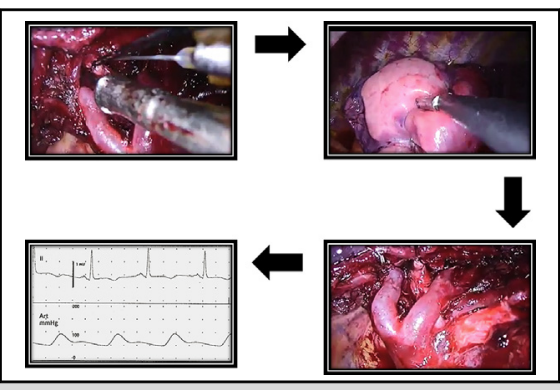

Air embolism, AMI, and cardiac arrest after air insufflation during VATS segmentectomy.

\section{Central Message}

We should be cautious when adopting the technique of selective segmental inflation with butterfly needles during VATS segmentectomy, as it can induce air embolism with cardiac arrest.

See Editorial Commentary page e115.
A 52-year-old woman, without any history of medical diseases, presented with ground-glass opacity in the anterior segment (LS3) of the left upper lung (Figure 1) incidentally found during a health examination. Her preoperative electrocardiogram (ECG), pulmonary function, and cardiac echography were normal. She underwent video-assisted thoracoscopic (VATS) left upper anterior segmentectomy. As planned, the segmental artery and intrasegmental vein to the anterior segment of the left upper lobe were identified and divided, and the segmental bronchus was looped and ligated with a silk tie. To recognize the intersegmental plane,

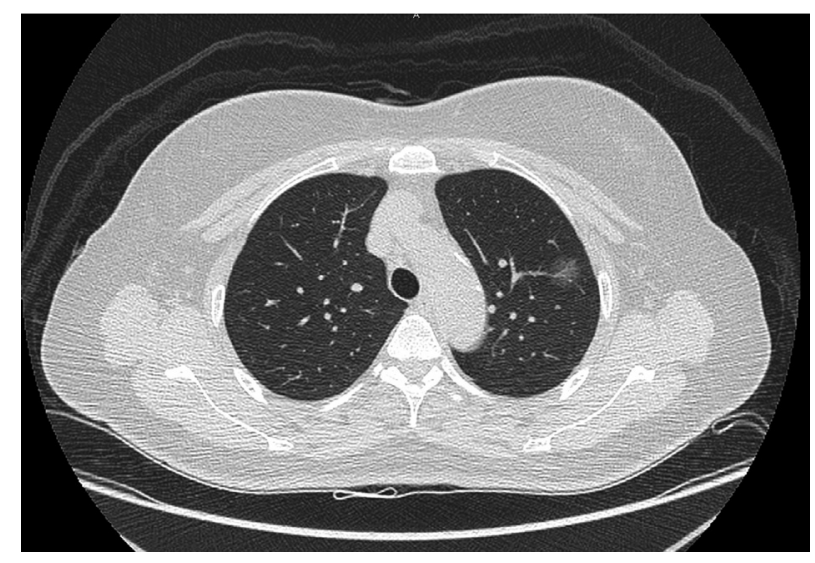

FIGURE 1. A 15 -mm ground-glass opacity that was confirmed to be lung adenocarcinoma was found on the low-dose computed tomography study.

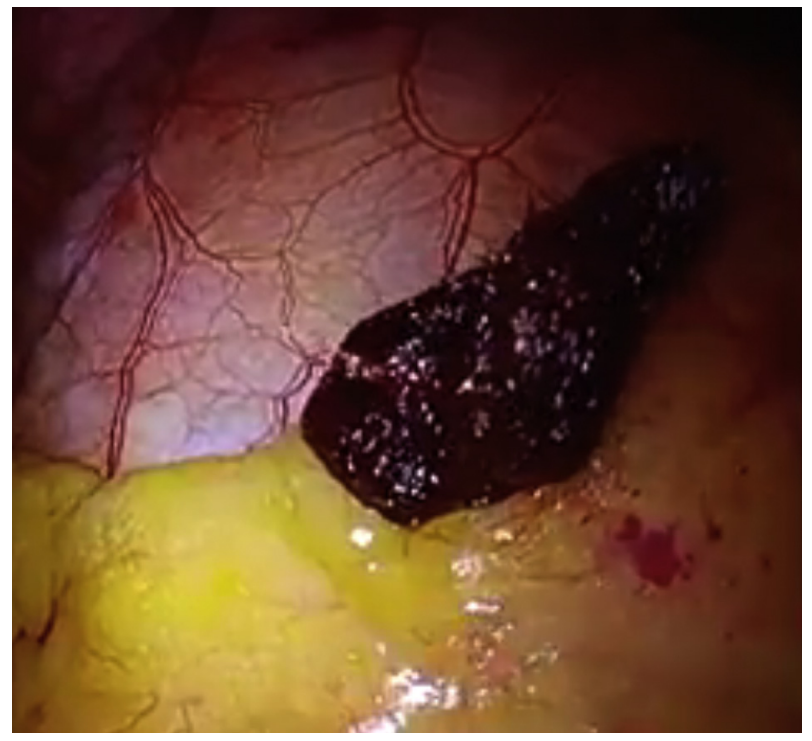

VIDEO 1. Air was instilled through a 23-gauge butterfly needle into the distal part of the segmental bronchus to inflate the target segment (1-20 seconds). Subsequently, concurrent bubble streaming in the intersegmental veins was noticed (21-34 seconds). Intracardiac injection of 1-mg epinephrine was administered at 54 seconds, and the heart resumed beating within 1 minute. Video available at: http://www.jtcvsonline.org/article/S0022-5223(17)326867/fulltext. 
$100 \mathrm{~mL}$ of air was injected into the distal part of the anterior segmental bronchus in the direction of the long axis of the bronchus through a 23-gauge butterfly needle after confirmation of negative blood regurgitation (Video 1).

Soon after the completion of injection of air, T-wave inversion (Figure 2, B) on the ECG followed by ST elevation (Figure 2, $C$-E), QRS widening (Figure 2, $C-E$ ), and hypotension (Figure 2, $C$-E) occurred. Subsequently, concurrent bubble streaming in the intersegmental veins was noticed by the surgeon (Video 1). Because of the patient's low blood pressure, extracorporeal cardiac massage was performed along with administration of 3 boluses of 1-mg intravenous epinephrine; however, the patient did not respond to these treatments, and her condition progressed to electromechanical dissociation with cardiac arrest (Figure 2, F).

Therefore, the surgeon administered $1 \mathrm{mg}$ of epinephrine directly into the left ventricular muscle (Video 1), and her heart resumed beating within 1 minute (Video 1). The entire episode lasted 6 minutes. After we ascertained that the patient was hemodynamically stable, the operation was recommenced and completed. The patient was then transferred to the intensive care unit with short-course mechanical ventilation with $100 \% \mathrm{O}_{2}$ therapy. Cardiac echography in the intensive care unit did not show the presence of air bubbles in the cardiac chambers but demonstrated apical septal hypokinesis and fair left ventricle ejection fraction of $58 \%$, which became completely normal on discharge at postoperative day 4 .

Findings of a neurologic evaluation revealed no symptoms/signs of brain infarct. Troponin I level was 40.5 $\mathrm{pg} / \mathrm{mL}$ (reference: $15.6 \mathrm{pg} / \mathrm{mL}$ ) during the episode, which increased to $1156.6 \mathrm{pg} / \mathrm{mL}$ in 2 hours and then reached the peak of $3261.8 \mathrm{pg} / \mathrm{mL} 20$ hours later. Postoperative serial ECG changes implying myocardial stunning showed global ST and marked T-wave abnormality with QT prolongation during postoperative days 1 to 4 . T-wave inversion in I, II,
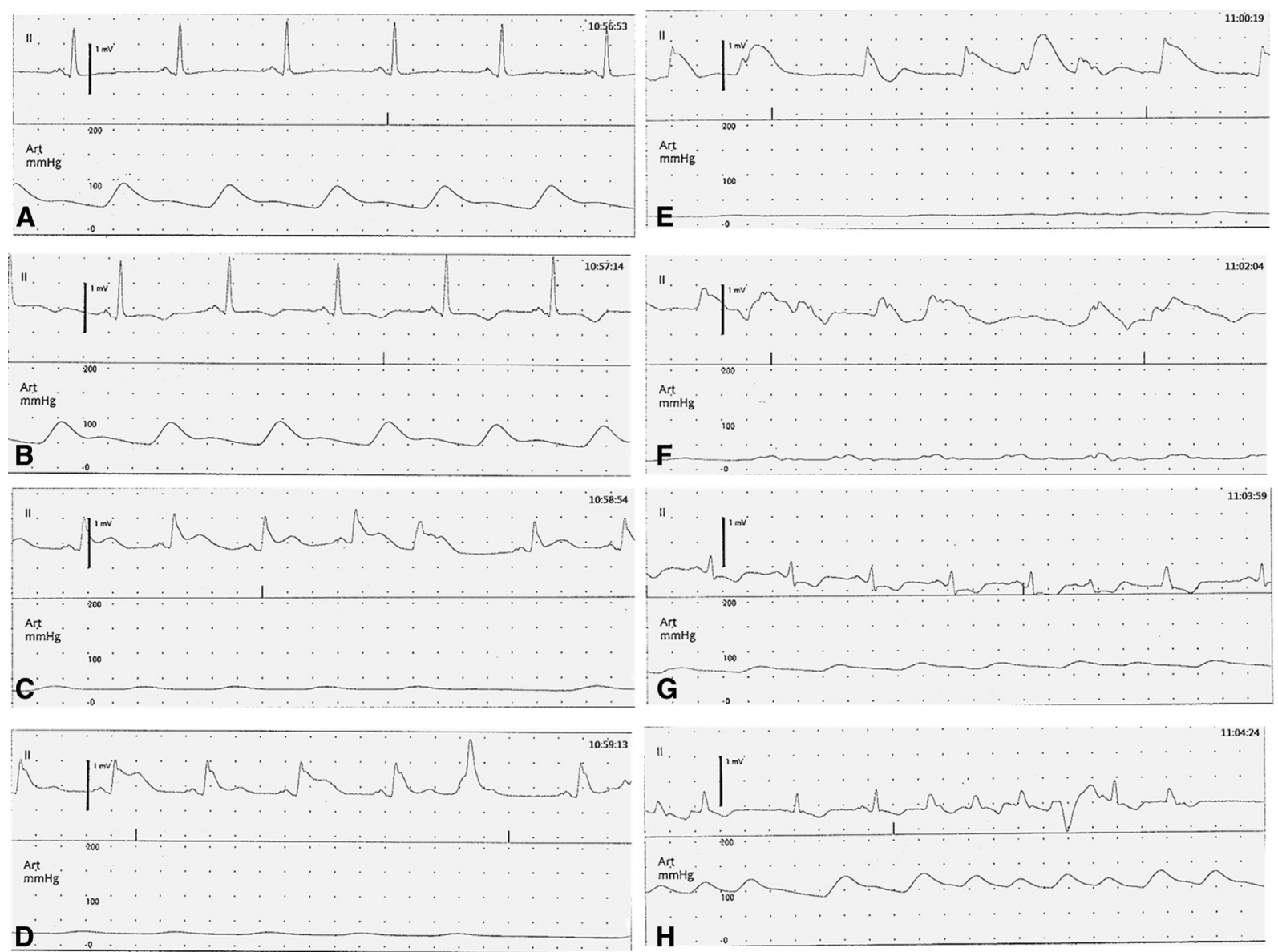

FIGURE 2. Serial changes of electrocardiogram (ECG) waveform and arterial blood pressure. A, Initial ECG and arterial blood pressure at the beginning of the air injection into the selected bronchus. B, T-wave inversion. C-E, ST elevation, QRS widening, and hypotension. Concurrent bubble streaming in the intersegmental veins was noticed. F, Electromechanical dissociation with cardiac arrest. One milligram of epinephrine was injected into the cardiac muscle. G-H, The heart resumed beating with marked ST-T changes and arrhythmia. 
aVL, aVF, and V2 to V6, although less prominent, persisted into postoperative day 10 up to 1 month. The patient returned to normal activity without any chest discomfort.

\section{DISCUSSION}

In all the previous reports on VATS segmentectomy, ${ }^{1,2}$ air embolisms caused brain infarction. Air embolism-induced acute myocardial ischemia and cardiac arrest during VATS segmentectomy have not been reported so far.

This patient had no risk factors for coronary artery disease. Therefore, the cause of acute myocardial ischemia was most likely to be inadvertent coronary air embolism, as the timing of the event was immediately after injection of air into the segmental bronchus and concurrent bubble streaming in the intersegmental vein. We postulated that a small amount of pressurized air injected into the lung parenchyma around the segmental bronchus leaked through the sharp tip of the butterfly needle. The air must have traversed the pulmonary microvasculature and reached the intersegmetnal vein and eventually reached the coronary arteries. A previous study ${ }^{3}$ showed that 0.5 to $1 \mathrm{~mL}$ of air injected into a pulmonary vein could cause cardiac arrest from coronary air embolism and ischemia. We postulated that most of the injected air went into the target segmental lung, as suggested by inflation of the lung after air injection; however, a small amount of air inadvertently entered the coronary arteries. Effective resuscitation, which had moved the air embolism distally, in addition to persistent high-flow oxygen administration, which had shrunk the size of air bubble, resulted in only subclinical ECG and enzyme changes; however, a smooth clinical course was achieved even after the cardiac arrest episode.

To detect the intersegmental plane, we adopted the selected segmental inflation technique, as it is more efficient than conventional inflation-deflation. Although we did not measure flow rate or pressure as recommended by Kamiyoshihara and colleagues, ${ }^{4}$ we used a syringe to confirm negative aspiration of blood as well as to inject air slowly with low resistance and ease of injection. Butterfly needles should be used with caution, as they bear the risk of air embolism. ${ }^{5}$ Although the surgeon kept the butterfly needle steady during injection, air leak through the tip of the needle might have caused the event (cardiac arrest). This is the first event we encountered among 32 cases treated using this technique. We no longer use butterfly needles; instead, small caliber nasogastric tubes are used during the selected segmental inflation technique.

\section{References}

1. Kiribayashi M, Nakasone M, Moriyama N, Mochida S, Yamasaki K, Minami Y, et al. Multiple cerebral infarction by air embolism associated with remarkable low BIS value during lung segmentectomy with video assisted thoracic surgery (VATS) technique: a case report (in Japanese). Masui. 2010;59:480-3.

2. Otsuka T, Nakamura Y, Harada A, Sato M. Extremely rare but potential complication of diffuse brain edema due to air embolism during lung segmentectomy with selected segmental inflation technique by syringe needle during video-assisted thoracoscopic surgery. J Thorac Cardiovasc Surg. 2011;142:e151-2.

3. Ho AM, Ling E. Systemic air embolism after lung trauma. Anesthesiology. 1999; 90:564-75.

4. Kamiyoshihara M, Nagashima T, Igai H. The selected segmental inflation technique for pulmonary segmentectomy: pros and cons. J Thorac Cardiovasc Surg. 2012;143:990-1.

5. Kamiyoshihara M, Kakegawa S, Morishita Y. Convenient and improved method to distinguish the intersegmental plane in pulmonary segmentectomy using a butterfly needle. Ann Thorac Surg. 2007;83:1913-4. 